\title{
Descriptor Representations without Direct Feedthrough Term*
}

\author{
M. KUIJPER $\dagger$
}

Key Words-Linear systems; minimal realization; multivariable systems; redundancy; state space.

\begin{abstract}
Descriptor representations are considered that are given by $(E, A, B, C, D)$ with $D=0$. Minimality under external equivalence is characterized in terms of the matrices $E, A, B$ and $C$. Also, transformations are given by which minimal $(E, A, B, C)$ representations are related under external equivalence. The transformations turn out to be more simple than in the " $D \neq 0$ " case. Algorithms for rewriting an $(E, A, B, C, D)$ representation in $(E, A, B, C)$ form are also given. Finally, a realization procedure is presented for obtaining a minimal $(E, A, B, C)$ representation for a system that is given in polynomial matrix fractional form.
\end{abstract}

1. Introduction and preliminaries

IN THIS PAPER we consider linear time invariant systems represented by

$$
\begin{aligned}
\sigma E \xi & =A \xi+B u, \\
y & =C \xi .
\end{aligned}
$$

Here the variables $y, u$ and $\xi$ are functions of time $(t \in \mathbb{T})$ that take values in the output space $Y$, the input space $U$ and the descriptor space $X_{d}$, respectively. Further, $\sigma$ denotes differentiation or shift, depending on whether one works in continuous time $\left(\mathbb{T}=\mathbb{R}_{+}\right)$or discrete time $\left(\mathbb{T}=\mathbb{Z}_{+}\right)$. The codomain of the mappings $E$ and $A$ will be denoted by $X_{e}$ (equation space). The matrices $E$ and $A$ are not assumed to be square.

The above representation is a specific form of the so-called descriptor representation

$$
\begin{aligned}
\sigma E \xi & =A \xi+B u, \\
y & =C \xi+D u .
\end{aligned}
$$

The only difference between (1.1) and (1.2) is the absence of a direct feedthrough term $D$ in (1.1). Unlike the standard state space case $(E=I)$, one here has a choice to consider either $(E, A, B, C)$ or $(E, A, B, C, D)$ representations. Indeed, $(E, A, B, C, D)$ representations can be rewritten in $(E, A, B, C)$ form and vice versa, as we will see in Section 3 of this paper. In some situations $(E, A, B, C)$ representations are preferred: two-point boundary-value descriptor systems (Nikhoukhah et al., 1987) are usually written in $(E, A, B, C)$ form because of the time-reversible character of such systems: a symmetric representation is preferred to a non-symmetric one. However, the absence of a $D$ term usually leads to a larger descriptor state space: the direct feedthrough term is related to the "non-dynamic" variables

* Received 7 January 1991; revised 3 July 1991; received in final form 13 September 1991. The original version of this paper was not presented at any IFAC meeting. This paper was recommended for publication in revised form by Associate Editor V. Kučera under the direction of Editor $\mathbf{H}$. Kwakernaak.

† CWI, P.O. Box 4079, 1009 AB Amsterdam, The Netherlands. of the system, as was first pointed out in Verghese et al. (1981).

The aim of this paper is the following. First, we consider the question under which conditions an $(E, A, B, C)$ representation of a system is minimal among all other equivalent $(E, A, B, C)$ representations. Here a descriptor representation is defined to be minimal if the rank of $E$, the column defect of $E$ (dimker $E$ ) and the row defect of $E$ (codim in $E$ ) are minimal. We will give a characterization of minimality in terms of the matrices $E, A, B$ and $C$. We will also give the complete set of transformations by which minimal equivalent $(E, A, B, C)$ representations can be transformed into each other. Finally, we show how a minimal $(E, A, B, C)$ realization can be constructed, starting from a system description in polynomial matrix fractional form.

The equivalence concept that we will use throughout the paper is that of so-called "external equivalence". Systems are called externally equivalent if their induced "behaviours" are the same. Here the behaviour of a system consists of the time trajectories of the input and output variables (the "external variables") that arise from the system representation. For more details and motivation the reader is referred to (Willems, 1983, 1986; Kuijper and Schumacher, 1990b). It should be noted that the set of time trajectories of the output variables that are not influenced by the input variables (the "uncontrolled behaviour") remains invariant under external equivalence. This constitutes one of the main differences between external equivalence and so-called transfer equivalence where the invariant is the transfer function instead of the behaviour.

In the development below, a prominent role is played by the so-called pencil representation:

$$
\begin{aligned}
\sigma G z & =F z, \\
y & =H_{y} z, \\
u & =H_{u} z .
\end{aligned}
$$

Here $F$ and $G$ are linear mappings from $Z$ to $X$, where $Z$ is the space of internal variables and $X$ is the equation space. It is shown in Willems (1986) and Kuijper and Schumacher (1990a) that a minimal pencil representation can be realized directly from the behaviour of the system in a natural way. Here the pencil representation is called minimal if both $\operatorname{dim} Z$ and $\operatorname{dim} X$ are minimal. In the next section we present algorithms for rewriting a pencil representation in $(E, A, B, C)$ form and vice versa. These algorithms are used for deriving minimality results and results on the transformation group in Section 3. In Section 4 we will give a procedure for realization into $(E, A, B, C)$ form. The procedure will be illustrated by an example.

\section{Relation with pencil form}

The next algorithm gives a procedure for rewriting a pencil representation in $(E, A, B, C)$ form.

Algorithm. 1. Let a pencil representation be given by $\left(F, G, H_{y}, H_{u}\right)$. Decompose the internal variable space $Z$ as 
$Z_{0} \oplus Z_{1} \oplus Z_{2} \oplus Z_{3}$ where $Z_{3}=\operatorname{ker} G \cap \operatorname{ker} H, \quad Z_{2} \oplus Z_{3}=$ ker $G \cap$ ker $H_{y}, Z_{1} \oplus Z_{2} \oplus Z_{3}=\operatorname{ker} G$. Accordingly, write

$$
\begin{array}{r}
G=\left[\begin{array}{llll}
G_{0} & 0 & 0 & 0
\end{array}\right], \quad F=\left[\begin{array}{lllll}
F_{0} & F_{1} & F_{2} & F_{3}
\end{array}\right], \\
H_{y}=\left[\begin{array}{llllll}
H_{y 0} & H_{y 1} & 0 & 0
\end{array}\right], \quad H_{u}=\left[\begin{array}{lllll}
H_{u 0} & H_{u 1} & H_{u 2} & 0
\end{array}\right] .
\end{array}
$$

Then the matrices $G_{0}$ and $H_{y 1}$ have full column rank. Also, the matrix $H_{u 2}$ has full column rank, and by renumbering the $u$-variables if necessary, we can write

$$
H_{u 0}=\left[\begin{array}{l}
H_{10} \\
H_{20}
\end{array}\right], \quad H_{u 1}=\left[\begin{array}{c}
H_{11} \\
H_{21}
\end{array}\right], \quad H_{u 2}=\left[\begin{array}{c}
H_{12} \\
H_{22}
\end{array}\right],
$$

where $H_{22}$ is invertible (or empty, if $\operatorname{ker} G \cap \operatorname{ker} H_{y} \subset$ ker $\left.H_{u}\right)$. Define descriptor matrices by

$$
\begin{array}{cc}
E=\left[\begin{array}{ccc}
G_{0} & 0 & 0 \\
0 & 0 & 0
\end{array}\right], \quad A=\left[\begin{array}{ccc}
\bar{F}_{0} & \bar{F}_{1} & F_{3} \\
\bar{H}_{10} & \bar{H}_{11} & 0
\end{array}\right], \quad B=\left[\begin{array}{cc}
0 & \bar{F}_{2} \\
-I & \bar{H}_{12}
\end{array}\right], \\
C=\left[\begin{array}{lll}
H_{y 0} & H_{y 1} & 0
\end{array}\right],
\end{array}
$$

with

$$
\begin{aligned}
\bar{F}_{0} & =F_{0}-F_{2} H_{22}^{-1} H_{20}, \\
\bar{F}_{1} & =F_{1}-F_{2} H_{22}^{-1} H_{21}, \\
\bar{H}_{10} & =H_{10}-H_{12} H_{22}^{-1} H_{20}, \\
\bar{H}_{11} & =H_{11}-H_{12} H_{22}^{-1} H_{21}, \\
\bar{F}_{2} & =F_{2} H_{22}^{-1}, \\
\bar{H}_{12} & =H_{12} H_{22}^{-1} .
\end{aligned}
$$

Vice versa, an $(E, A, B, C)$ representation can be rewritten in pencil form by applying the algorithm of Kuijper and Schumacher (1990b) which transforms $(E, A, B, C, D)$ representations to equivalent pencil representations. In Kuijper and Schumacher (1990b) it is shown that this algorithm preserves minimality: minimal $(E, A, B, C, D)$ representations are transformed to minimal pencil representations. Surprisingly, the algorithm (that will be called Algorithm 2 in the sequel) also preserves minimality for $(E, A, B, C)$ representations, as will be shown later.

We will now prove that applying Algorithm 1 to a minimal pencil representation leads to an externally equivalent $(E, A, B, C)$ representation that is minimal. For this purpose we first have to explore the concept of minimality under external equivalence for $(E, A, B, C)$ representations. In the following we will present lower bounds for some of the indices that have to be minimized. These involve a certain invariant subspace $W^{0} \subset W(=Y \oplus U)$ (see Willems, 1986; Kuijper and Schumacher, 1990a). Intuitively speaking, the subspace $W^{0}$ is spanned by the minimum number of "driving variables" of the system; when $W^{0}$ coincides with the input space we are dealing with a system with a strictly causal input-output structure. A definition of $W^{0}$ will be given in Section 4 . In the following lemma $W^{0}$ is expressed in terms of the matrices $E, A, B$ and $C$. The mapping $\pi_{Y}: W \rightarrow Y$ denotes projection onto $Y$ along $U$.

Lemma 2.1. Let a descriptor representation be given by $(E, A, B, C)$. Then necessary conditions for $(E, A, B, C)$ to be minimal under external equivalence are

(i) $\left[\begin{array}{ll}E & B\end{array}\right]$ is surjective,

(ii) $\left[E^{T} C^{T}\right]^{T}$ is injective.

Moreover, if (i) and (ii) hold we have

$$
\begin{aligned}
U \cap W^{0} & =B^{-1}[\operatorname{im} E], \\
\pi_{Y} W^{0} & =C[\operatorname{ker} E] .
\end{aligned}
$$

Proof. The fact that conditions (i) and (ii) are necessary for minimality is proved in Kuijper and Schumacher (1990a): Lemma 7.2 and Lemma 7.3 of that paper are also valid for $(E, A, B, C)$ representations. The other statements follow from arguments that are analogous to those in the proof of Lemma 3 of Kuijper and Schumacher (1990b).

From the proofs of Lemma 7.2 and Lemma 7.3 in Kuijper and Schumacher (1990a) we immediately have the following corollary.
Corollary 2.2. Let a descriptor representation be given by $(E, A, B, C)$. Then we have

(i) $\operatorname{dim} \operatorname{ker} E \geqslant \operatorname{dim}\left(\pi_{Y} W^{0}\right)$,

(ii) $\operatorname{codim}$ im $E \geqslant \operatorname{codim}\left(U \cap W^{0}\right)$.

We are now ready for the main theorems of this section.

Theorem 2.3. Let $(E, A, B, C)$ be a descriptor representation that results from applying Algorithm 1 to a pencil representation, given by $\left(F, G, H_{y}, H_{u}\right)$. Then the two representations are externally equivalent. Furthermore if $\left(F, G, H_{y}, H_{u}\right)$ is minimal then $(E, A, B, C)$ is also minimal.

Proof. The external equivalence of the two representations follows as in the proof of Lemma 1 in Kuijper and Schumacher (1990b). Further, the minimality of $\left(F, G, H_{y}, H_{u}\right)$ implies that rank $G$ is minimal. The minimality of rank $E$ now follows immediately since in both Algorithm 1 and Algorithm 2 we have that rank $E=\operatorname{rank} G$. The minimality of $\left(F, G, H_{y}, H_{u}\right)$ also implies that $G$ is surjective and that $\left[G^{T} H_{y}^{T^{T}} H_{u}^{T}\right]^{T}$ is injective (Proposition 1.1 in Kuijper and Schumacher (1990a)) from which it follows that $\left[\begin{array}{ll}E & B\end{array}\right]$ is surjective and $\left[E^{T} C^{T}\right]^{T}$ is injective $\left(Z_{3}=\{0\}\right)$. From Lemma 2.1 it now follows that the lower bounds in Corollary 2.2 are reached so that we can conclude that the representation $(E, A, B, C)$ is minimal under external equivalence.

Theorem 2.4. Let $\left(F, G, H_{y}, H_{u}\right)$ be a pencil representation that results from applying Algorithm 2 to an $(E, A, B, C)$ representation. Then the two representations are externally equivalent. Furthermore if $(E, A, B, C)$ is minimal then $\left(F, G, H_{y}, H_{u}\right)$ is also minimal.

Proof. The external equivalence of the representations has been proven in Lemma 2 of Kuijper and Schumacher (1990b). Next, by Lemma 2.1, the minimality of $(E, A, B, C)$ implies that $\left[\begin{array}{ll}E & B\end{array}\right]$ is surjective. It can then be concluded that $G$ is surjective. Furthermore the minimality of rank $G$ follows as in the proof of the previous theorem. Finally we have

$$
\begin{aligned}
\operatorname{dim} \operatorname{ker} G & =\operatorname{dim} \operatorname{ker} E+\operatorname{dim} B^{-1}[\operatorname{im} E], \\
& =\operatorname{dim}\left(\pi_{Y} W^{0}\right)+\operatorname{dim}\left(U \cap W^{0}\right), \\
& =\operatorname{dim} W^{0} .
\end{aligned}
$$

From Kuijper and Schumacher (1990a) we may now conclude that $(F, G, H)$ is minimal.

\section{Minimality and the transformation group}

Theorem 3.1. Let a descriptor representation be given by $(E, A, B, C)$. The representation is minimal under external equivalence if and only if the following conditions hold:

(i) $\left[\begin{array}{ll}E & B\end{array}\right]$ is surjective,

(ii) $\left[E^{T} C^{T}\right]^{T}$ is injective,

(iii) $\left[s E^{T}-A^{T} C^{T}\right]^{T}$ has full column rank for all $s \in \mathbb{C}$.

Proof. From Lemma 2.1 it follows immediately that conditions (i) and (ii) should hold. In order to prove (iii) we apply Algorithm 2 to the representation. According to Theorem 2.4 the pencil representation $\left(F, G, H_{y}, H_{u}\right)$ that is obtained in this way is minimal. This implies that $\left[\begin{array}{lll}s G^{T}-F^{T} & H_{y}^{T} & H_{u}^{T}\end{array}\right]^{T}$ should have full column rank for all $s \in \mathbb{C}$ (Proposition 1.1 in Kuijper and Schumacher (1990a)). It is then easily seen that condition (iii) should hold. Conversely, when Algorithm 2 is applied to an $(E, A, B, C)$ representation for which conditions (i)-(iii) hold, it is easily seen that the resulting pencil representation satisfies the conditions of Proposition 1.1 in Kuijper and Schumacher $(1990 \mathrm{a})$ and is therefore minimal. From this it follows that rank $E$ is minimal. Furthermore since conditions (i) and (ii) are assumed to be satisfied we can use Lemma 2.1 to derive

and

$$
\operatorname{dim} \operatorname{ker} E(=\operatorname{dim} C[\operatorname{ker} E])=\operatorname{dim} \pi_{Y} W^{0},
$$

$\operatorname{codim} \operatorname{im} E\left(=\operatorname{codim} B^{-1}[\operatorname{im} E]\right)=\operatorname{codim} U \cap W^{0}$.

By Corollary 2.2 this proves that the $(E, A, B, C)$ representation is minimal. 
Remark 3.2. In the above theorem there is no requirement on the absence of non-dynamic variables $(A[\operatorname{ker} E] \subset \operatorname{im} E)$ as in the analogous theorem for $(E, A, B, C, D)$ representations (Theorem 4 in Kuijper and Schumacher (1990b)). This is not surprising since for $(E, A, B, C)$ representations the nondynamic variables cannot be eliminated: there is no $D$ term in which they can be incorporated.

Next, we present two algorithms that will clarify the relation between $(E, A, B, C)$ and $(E, A, B, C, D)$ representations. The first algorithm gives a procedure for rewriting an $(E, A, B, C, D)$ representation in $(E, A, B, C)$ form while the reverse procedure is given by the second algorithm

Algorithm 3. Let a descriptor representation be given by $(E, A, B, C, D)$. Let $V=\left[\begin{array}{ll}V_{1} & V_{2}\end{array}\right]$ be an invertible matrix such that

$$
D V=D\left[\begin{array}{ll}
V_{1} & V_{2}
\end{array}\right]=\left[\begin{array}{ll}
D_{1} & 0
\end{array}\right],
$$

where $D_{1}$ is injective. Let $T=\left[\begin{array}{ll}T_{1}^{T} & T_{2}^{T}\end{array}\right]^{T}$ be the inverse of $V$. Define $\xi_{2}=T_{1} u$. Then $D u=D_{1} \xi_{2}$. Now define a representation $(\tilde{E}, \tilde{A}, \tilde{B}, \tilde{C})$ by

$$
\tilde{E}=\left[\begin{array}{ll}
E & 0 \\
0 & 0
\end{array}\right], \quad \tilde{A}=\left[\begin{array}{cc}
A & 0 \\
0 & I
\end{array}\right], \quad \tilde{B}=\left[\begin{array}{c}
B \\
-T_{1}
\end{array}\right], \quad \tilde{C}=\left[\begin{array}{ll}
C & D_{1}
\end{array}\right],
$$

Theorem 3.3. Let $(\tilde{E}, \tilde{A}, \bar{B}, \tilde{C})$ be a descriptor representation that results from applying Algorithm 3 to an $(E, A, B, C, D)$ representation. Then the two representations are externally equivalent.

Proof. The operations that are involved in the algorithm clearly do not affect the behaviour: variables are merely written in another way. From this the external equivalence of the two representations follows.

Algorithm 4. Let a descriptor representation be given by $(E, A, B, C)$. Decompose the descriptor space $X_{d}$ as $X_{d 1} \oplus X_{d 2} \oplus X_{d 3}$ where $X_{d 3}=A^{-1}[\operatorname{im} E] \cap \operatorname{ker} E$ and $X_{d 2} \oplus$ $X_{d 3}=\operatorname{ker} E$. Decompose the equation space $X_{e}$ as $X_{e 1} \oplus X_{e 2}$ where $X_{e 1}=\operatorname{im} E$. Accordingly write

$$
\begin{gathered}
E=\left[\begin{array}{lll}
I & 0 & 0 \\
0 & 0 & 0
\end{array}\right], \quad A=\left[\begin{array}{lll}
A_{11} & A_{12} & A_{13} \\
A_{21} & A_{22} & 0
\end{array}\right], \\
B=\left[\begin{array}{l}
B_{1} \\
B_{2}
\end{array}\right], \quad C=\left[\begin{array}{lll}
C_{1} & C_{2} & C_{3}
\end{array}\right] .
\end{gathered}
$$

Then the matrix $A_{22}$ is injective. Choose an appropriate basis in $X_{e 2}$, such that w.r.t. this basis we have

$$
A_{21}=\left[\begin{array}{l}
A_{211} \\
A_{212}
\end{array}\right], \quad A_{22}=\left[\begin{array}{l}
1 \\
0
\end{array}\right], \quad B_{2}=\left[\begin{array}{l}
B_{21} \\
B_{22}
\end{array}\right] .
$$

Now define a representation $(\tilde{E}, \tilde{A}, \tilde{B}, \tilde{C}, \tilde{D})$ by

$$
\begin{gathered}
\tilde{E}=\left[\begin{array}{ll}
I & 0 \\
0 & 0
\end{array}\right], \quad \tilde{A}=\left[\begin{array}{cc}
A_{11}-A_{12} A_{211} & A_{13} \\
A_{212} & 0
\end{array}\right] \\
\tilde{B}=\left[\begin{array}{c}
B_{1}-A_{12} B_{21} \\
B_{22}
\end{array}\right], \quad \tilde{C}=\left[\begin{array}{ll}
C_{1}-C_{2} A_{211} & C_{3}
\end{array}\right], \quad \tilde{D}=-C_{2} B_{21} .
\end{gathered}
$$

Theorem 3.4. Let $(\bar{E}, \bar{A}, \bar{B}, \bar{C}, \bar{D})$ be a descriptor representation that results from applying Algorithm 4 to an $(E, A, B, C)$ representation. Then the two representations are externally equivalent. Furthermore if $(E, A, B, C)$ is minimal then $(\tilde{E}, \tilde{A}, \tilde{B}, \bar{C}, \bar{D})$ is also minimal.

Proof. The external equivalence of the two representations follows as in the proof of Lemma 1 in Kuijper and Schumacher $(1990 \mathrm{~b})$. The preservation of minimality is proven by using Theorem 3.1.

Next, we ask ourselves by which transformations minimal equivalent $(E, A, B, C)$ representations are related. The next theorem involves the concept of restricted system equivalence which was defined by Rosenbrock (1974).
Theorem 3.5. Let $(E, A, B, C)$ and $(\tilde{E}, \tilde{A}, \tilde{B}, \tilde{C})$ be descriptor representations that are minimal under external equivalence. Then the two representations are externally equivalent if and only if they are restricted system equivalent, i.e. there exist invertible matrices $M$ and $N$ such that

$$
\left[\begin{array}{cc}
M & 0 \\
0 & I
\end{array}\right]\left[\begin{array}{cc}
s E-A & -B \\
C & 0
\end{array}\right]=\left[\begin{array}{cc}
s \tilde{E}-\tilde{A} & -\tilde{B} \\
\tilde{C} & 0
\end{array}\right]\left[\begin{array}{cc}
N & 0 \\
0 & I
\end{array}\right]
$$

Proof. The proof is analogous to the proof of Theorem 5 of Kuijper and Schumacher (1990b). The input space $U$ should now be decomposed in a different way, namely as $U \cap W^{0} \oplus U_{2}$. Using the notation of the proof of Theorem 5 of Kuijper and Schumacher (1990b) we now have $D_{2}=0$, $\tilde{D}_{2}=0$ and (resulting from the fact that $\tilde{C}_{2}$ is injective) $T_{6}=0$. This implies that $X=0$ and $Y=0$, i.e. that the representations are restricted system equivalent.

Remark 3.6. The above theorem tells us that for minimal ( $E, A, B, C$ ) representations the only operations that are allowed under external equivalence are "change of basis" in $X_{d}$ and in $X_{e}$. Therefore the situation for $(E, A, B, C)$ representations parallels the standard state space case: minimal equivalent representations are related by "similarity". Note that this does not hold for minimal $(E, A, B, C, D)$ representations: they are related by operations of strong equivalence (Theorem 5 in Kuijper and Schumacher (1990b)).

\section{A realization procedure}

In Kuijper and Schumacher (1990a) we presented a method for obtaining a minimal $(E, A, B, C, D)$ representation for a system described by equations of the form

$$
R_{1}(\sigma) y+R_{2}(\sigma) u=0,
$$

where $R_{1}(s)$ and $R_{2}(s)$ are polynomial matrices of sizes $k \times p$ and $k \times m$, respectively. In this section we will indicate what changes should be made in the procedure in order to arrive at a (minimal) $(E, A, B, C)$ representation. We will use the notation of Section 8 of Kuijper and Schumacher (1990a).

As in Kuijper and Schumacher (1990a) we may assume that $\left[R_{1}(s) R_{2}(s)\right]$ is row proper. This means that we can write

$$
\left[R_{1}(s) \quad R_{2}(s)\right]=\Delta(s) B(s),
$$

where $B(s)=\left[B_{1}(s) B_{2}(s)\right]$ is right bicausal, and $\Delta(s)=$ $\operatorname{diag}\left(s^{\kappa_{1}}, \ldots, s^{\kappa_{k}}\right)$. We note here that $\left[B_{1}(\infty) B_{2}(\infty)\right]$ is the "leading row coefficient matrix" of $\left[R_{1}(s) R_{2}(s)\right]$ and that the subspace $W^{0}$ can be defined as Kuijper and Schumacher (1990a)

$$
W^{0}=\operatorname{ker}\left[B_{1}(\infty) \quad B_{2}(\infty)\right]
$$

In the realization method of Kuijper and Schumacher (1990a) a certain choice is made for a constant matrix $\bar{B}$ such that $\hat{B}(s)=\left[\begin{array}{lll}B^{T}(s) & \tilde{B}^{T}\end{array}\right]^{T}$ is bicausal. It was shown that this choice naturally leads to a realization in $(E, A, B, C, D)$ form. Here our choice of $B$ has to be different since our objective is to construct an $(E, A, B, C)$ realization. First note that we have

$$
U \cap W^{0}=\operatorname{ker} B_{2}(\infty)
$$

This enables us to decide which $u$-variables are driving variables. By renumbering the inputs if necessary, we may assume that

$$
B_{2}(\infty)=\left[\begin{array}{ll}
B_{2}^{1}(\infty) & B_{2}^{2}(\infty)
\end{array}\right]
$$

where $B_{2}^{1}(\infty)$ has full column rank, and the columns of $B_{2}^{2}(\infty)$ depend linearly on those of $B_{2}^{1}(\infty)$. Let $B_{2}^{2}(\infty)$ have $m_{2}$ columns; note that $m_{2} \leqslant p+m-k$ and that we have $m_{2}=0$ when $\operatorname{dim} \operatorname{ker} B_{2}(\infty)=0$. It is easily verified that a matrix $\tilde{B}$ which completes $B(\infty)$ to an invertible matrix may be found 

of the form $\left(\begin{array}{lll}\bullet & 0 & 0 \\ 0 & 0 & I\end{array}\right)$. We then have that $\hat{B}(\infty)^{-1}$ is of the
form

$$
\hat{B}(\infty)^{-1}=\left(\begin{array}{ccc}
* & * & 0 \\
* & * & * \\
0 & 0 & I
\end{array}\right)
$$

where the partitioning is $\left(p+m_{1}+m_{2}\right) \times\left(k+\left(p+m_{1}-\right.\right.$ $\left.k)+m_{2}\right)\left(m_{1}\right.$ is the number of columns of $\left.B_{2}^{1}(\infty)\right)$. The realization procedure of Kuijper and Schumacher (1990a) then leads to equations of the following form:

$$
\begin{aligned}
\sigma z_{0} & =A_{0} z_{0}+B_{1} z_{1}+B_{2} z_{2}, \\
y & =H_{00} z_{0}+H_{01} z_{1}, \\
u_{1} & =H_{10} z_{0}+H_{11} z_{1}+H_{12} z_{2}, \\
u_{2} & =z_{2} .
\end{aligned}
$$

This can obviously be rewritten in $(E, A, B, C)$ form:

$$
\begin{aligned}
\sigma\left(\begin{array}{ll}
I & 0 \\
0 & 0
\end{array}\right)\left(\begin{array}{l}
z_{0} \\
z_{1}
\end{array}\right) & =\left(\begin{array}{cc}
A_{0} & B_{1} \\
H_{10} & H_{11}
\end{array}\right)\left(\begin{array}{l}
z_{0} \\
z_{1}
\end{array}\right)+\left(\begin{array}{cc}
0 & B_{2} \\
-I & H_{12}
\end{array}\right)\left(\begin{array}{l}
u_{1} \\
u_{2}
\end{array}\right), \\
y & =\left[\begin{array}{ll}
H_{(1)} & H_{01}
\end{array}\right]\left(\begin{array}{l}
z_{0} \\
z_{1}
\end{array}\right) .
\end{aligned}
$$

Here rank $E=n$ where $n$ denotes the sum of the minimal row indices $\left(\kappa_{i}\right)$ of $\left[R_{1}(s) R_{2}(s)\right]$. As in Kuijper and Schumacher $(1990 \mathrm{a})$ it can be verified that the above $(E, A, B, C)$ realization is minimal.

Remark 4.1. Let us assume that $R_{1}(s)$ and $R_{2}(s)$ are left coprime so that we can compare our realization procedure with procedures under transfer equivalence, such as in Wimmer (1981) and Conte and Perdon (1982). We are then essentially starting from a transfer function $T(s)=$ $R_{1}^{-1}(s) R_{2}(s)$ given as a polynomial left coprime factorization. In both Wimmer (1981) and Conte and Perdon (1982) a minimal $(E, A, B, C)$ representation is obtained by splitting the finite and infinite frequencies. In the resulting $(E, A, B, C)$ representation the matrix $E$ has the form $\left(\begin{array}{ll}I & 0 \\ 0 & 0\end{array}\right)$ (the matrix $A$ has the form $\left(\begin{array}{ll}* & 0 \\ 0 & I\end{array}\right)$ ). In contrast, our procedure does not split finite and infinite frequencies (leading to a matrix $E$ of the form $\left(\begin{array}{ll}I & 0 \\ 0 & 0\end{array}\right)$ ) thus providing a more direct link between polynomial and state space representations. This makes it easier to translate system properties from polynomial terms to state space terms and vice versa. Note that it follows from Theorem 3.5 that the two representations are restricted system equivalent.

We conclude this section with an example.

Example 4.2. Take

$\left[R_{1}(s) \quad R_{2}(s)\right]=\left(\begin{array}{cccccc}s+2 & 0 & s+1 & 0 & 1 & 0 \\ 0 & s-1 & 0 & 3 & s & s+4 \\ s & 0 & 0 & s^{2} & 0 & 0\end{array}\right)$,

corresponding to three outputs and three inputs. The leading row coefficient matrix

$$
\left(\begin{array}{llllll}
1 & 0 & 1 & 0 & 0 & 0 \\
0 & 1 & 0 & 0 & 1 & 1 \\
0 & 0 & 0 & 1 & 0 & 0
\end{array}\right)
$$

has fuli row rank, so that the given matrix $R(s)$ is already row reduced; also $m_{2}=1$ and the inputs need not be renumbered. We see that the sum of the minimal row indices of $R(s)$ is four and that the rank of $B_{2}(\infty)$ (formed by the last three columns of the matrix above) is two; so, a descriptor representation $(E, A, B, C)$ will be minimal if and only if the matrix $E$ has size $6 \times 6$ and rank 4 .

Applying the above procedure we take

$$
\tilde{B}=\left(\begin{array}{llllll}
1 & 0 & 0 & 0 & 0 & 0 \\
0 & 1 & 0 & 0 & 0 & 0 \\
0 & 0 & 0 & 0 & 0 & 1
\end{array}\right)
$$

which leads to

$$
\hat{B}(\infty)^{1}=\left(\begin{array}{rrrrrr}
0 & 0 & 0 & 1 & 0 & 0 \\
0 & 0 & 0 & 0 & 1 & 0 \\
1 & 0 & 0 & -1 & 0 & 0 \\
0 & 0 & 1 & 0 & 0 & 0 \\
0 & 1 & 0 & 0 & -1 & -1 \\
0 & 0 & 0 & 0 & 0 & 1
\end{array}\right) .
$$

Consequently, we get the $(E, A, B, C)$ realization

$$
\begin{gathered}
\sigma\left(\begin{array}{lll}
I_{4} & 0 & 0 \\
0 & 0 & 0 \\
0 & 0 & 0
\end{array}\right)\left(\begin{array}{l}
z_{1} \\
z_{1} \\
z_{2}
\end{array}\right)=\left(\begin{array}{rrrrrr}
-1 & -1 & 0 & 0 & -1 & 1 \\
0 & 0 & -3 & 0 & 0 & 1 \\
0 & 0 & 0 & 1 & -1 & 0 \\
0 & 0 & 0 & 0 & 0 & 0 \\
0 & 0 & 1 & 0 & 0 & 0 \\
0 & 1 & 0 & 0 & 0 & -1
\end{array}\right) \\
x\left(\begin{array}{l}
z_{11} \\
z_{1} \\
z_{2}
\end{array}\right)+\left(\begin{array}{rrrr}
0 & 0 & 1 \\
0 & 0 & -4 \\
0 & 0 & 0 \\
0 & 0 & 0 \\
-1 & 0 & 0 \\
0 & -1 & -1
\end{array}\right)\left(\begin{array}{l}
u_{1} \\
u_{2} \\
u_{1}
\end{array}\right) \\
y=\left(\begin{array}{llllll}
0 & 0 & 0 & 0 & 1 & 0 \\
0 & 0 & 0 & 0 & 0 & 1 \\
1 & 0 & 0 & 0 & -1 & 0
\end{array}\right)\left(\begin{array}{l}
z_{1} \\
z_{1} \\
z_{2}
\end{array}\right)
\end{gathered}
$$

Remark 4.3. In the above example the column defect of $B_{1}(\infty)$ is equal to 1. Therefore tsee Kuiper and Schumacher $(199($ a) ) a minimal $(E, A, B, C, I)$ realizatien would have an $E$-matrix of size $5 \times 5$ (and again rank 4 ). Indeed, there is one nondynamic variable in the above (E, $A, B$, () realization, namely the variable $z$, which can tre incorporated in a $D$ term.

\section{Conclusions}

In this paper we have characterized the mimimality of an $(E, A, B, C)$ representation in terms of the matrices $E, A, B$ and $C$ The conditions are the same as for $(f, A, B, C, D)$ representations, except that there is no regurement on the absence of non-dynamic modes.

We also gave a procedure for realizing systems given by autoregressive equations 16 minmal $(E, A, B, C)$ form. It is proven that such a realization is unique un to operations of restricted system equivalence. Thus the transformation group consists of isomorphisms and is therefore more simple than in the case of $(E, A, B,(, I))$ pepresentatuons in some situations this might be a reason to use an $(F, A, B,()$ representation rather than an $(E, A, B,(, I))$ representation

\section{References}

Conte, $G$ and A. M. Perdon (1982). (ieneralized state space realizations for non-proper rational transfer functions. Syst. Contr. L.ett., 1, 270 276

Kuijper, M. and J. M. Schumacher (I $\left.\%()_{i a}\right)$. Realization of autoregressive equations in pencil and descriptor form. SIAM J. Comtr. Optimiz, 28, 1162-1189

Kuijper, M. and J. M. Schumacher (19\%(b)). Minimality of descriptor representations under external equivalence. Automatica, 27, 985 945.

Nikoukhah, R., A. S. Willsky and B. ('. Levy (1987). Boundary-value descriptor systems: well-ponedness, reachability and observability. Int. J. (ontrol, 46, 1715-1737.

Rosenbrock, H. H. (1974). Structural properties of linear dynamical systems. Int. J. (omerol, 20, 191-202.

Verghese, (3., B. C. levy and I. Kanlath (1981). A 
generalized state space for singular systems. IEEE Trans. Aut. Control, AC-26, 811-831.

Willems, J. C. (1983). Input-output and state space representations of finite-dimensional linear time-invariant systems. Lin. Alg. Appl., 50, 581-608.
Willems, J. C. (1986). From time series to linear system. Part I: Finite-dimensional linear time invariant systems. Automatica, 22, 561-580.

Wimmer, H. K. (1981). The structure of non-singular polynomial matrices. Math. Systems Theory, 14, 367-379. 